\title{
Gradient nanostructured titanium stimulates cell responses in vitro and enhances osseointegration in vivo
}

\author{
Nan-Jue Cao ${ }^{1,2 \#}$, Yu-He Zhu ${ }^{1 \# \wedge}$, Fei Gao ${ }^{1}$, Chen Liang ${ }^{3}$, Zhen-Bo Wang $^{3}$, Yue Zhang ${ }^{1}$, Chun-Ping Hao ${ }^{1 \wedge}$, \\ Wei Wang ${ }^{1 \wedge}$ \\ ${ }^{1}$ School and Hospital of Stomatology, China Medical University, Liaoning Provincial Key Laboratory of Oral Diseases, Shenyang, China; ${ }^{2}$ The \\ Fourth Affiliated Hospital, Zhejiang University School of Medicine, Yiwu, China; ${ }^{3}$ Shenyang National Laboratory for Materials Science, Institute of \\ Metal Research, Chinese Academy of Sciences, Shenyang, China \\ Contributions: (I) Conception and design: W Wang, NJ Cao, YH Zhu; (II) Administrative support: W Wang; (III) Provision of study materials or \\ patients: C Liang, ZB Wang; (IV) Collection and assembly of data: NJ Cao, YH Zhu, F Gao; (V) Data analysis and interpretation: NJ Cao, YH Zhu, \\ F Gao, W Wang; (VI) Manuscript writing: All authors; (VII) Final approval of manuscript: All authors. \\ \#These authors contributed equally to this work. \\ Correspondence to: Prof. Wei Wang. School and Hospital of Stomatology, China Medical University, Liaoning Provincial Key Laboratory of Oral \\ Diseases, No. 117, Nanjing North Street, Heping District, Shenyang 110002, China. Email: wwang75@cmu.edu.cn.
}

Background: Though titanium (Ti) is widely used as dental materials in the clinic, effective methods to treat Ti for higher surface biological activity still lack. Through Surface mechanical attrition treatment (SMAT) technology we could endow Ti with gradient nanostructured surface (GNS Ti). To investigate the biocompatibility of GNS Ti for its further application in dental implant field, we study the effects of GNS Ti on cell responses in vitro and osseointegration of the implant with surrounding bone tissues in vivo.

Methods: In this study, GNS Ti was fabricated by SMAT. In vitro experiment, we co-cultured GNS Ti with bone mesenchymal stem cells (BMSCs), surface characterization was detected by transmission electron microscope (TEM). Adhesion, proliferation and differentiation of BMSCs were evaluated by scanning electron microscope (SEM), MTT, flow cytometry (FCM), alkaline phosphatase (ALP) and osteocalcin (OCN) tests. in vivo experiment, the GNS Ti was implanted into the rabbit mandible. Osteogenesis and osseointegration were evaluated by Micro CT, toluidine blue staining, and immunohistochemical staining at 4, 8 , and 12 weeks postoperatively.

Results: Both results showed that compared with the coarse grained (CG) Ti, the GNS Ti stimulated the adhesion, proliferation, and differentiation of BMSCs and improved osteogenesis and osseointegration.

Conclusions: This study indicates that gradient nanostructured Ti is a promising material for dental implant application.

Keywords: Surface mechanical attrition treatment (SMAT); titanium (Ti); nanostructure; bone mesenchymal stem cells (BMSCs); osseointegration

Submitted Nov 21, 2020. Accepted for publication Jan 22, 2021.

doi: $10.21037 /$ atm-20-7588

View this article at: http://dx.doi.org/10.21037/atm-20-7588

\footnotetext{
^ ORCID: Nan-Jue Cao, 0000-0001-6994-5924; Yu-He Zhu, 0000-0002-9771-0409; Chun-Ping Hao, 0000-0001-5258-2768; Wei Wang, 0000-0003-2798-0400.
} 


\section{Introduction}

With brilliant biocompatibility and mechanical properties, titanium (Ti) is widely used as dental implants and orthopedic implants in the clinic (1). However, studies have shown that $\mathrm{Ti}$, as an inert metal, has low surface biological activity, making it difficult to form stable osseointegration and may lead to bone resorption around the implant, periimplant inflammation, and even implant failure (2). Many bioengineers enhance the biological response of $\mathrm{Ti}$ and its alloys by coating them with hydroxyapatite (HA) (3) and $\mathrm{TiO}_{2}$ (4). Although the coating methods have achieved good biological results, the connection strength between the coating and the substrate is poor, which is prone to longterm clinical problems.

To avoid the risk of separation between the coating and the base metal and to enhance the osseointegration, Lu et al. (5) developed the surface mechanical attrition treatment (SMAT) technology. As a surface severe plastic deformation (SPD) technology, SMAT can realize the selfnanocrystallization on the material surface without changing the chemical composition. During the SMAT process, steel balls with a smooth surface, average size, and high-energy impact material surface repeatedly in a vacuum chamber. Plastic deformation in the material surface gradually makes the surface into the nano-sized grain and coarse grain to realize the nanoscale grain from the topmost surface to the internal micron level grain, namely characteristic of gradient nanostructured surface (GNS).

At present, SMAT technology has been successfully applied to a variety of metals, such as stainless steel (6), tungsten alloy (7), and magnesium alloy $(8,9)$. On the one hand, it is reported that the nanocrystalline layer obtained by SMAT improves mechanical properties such as hardness, abrasion resistance, ductility, and fatigue resistance $(6,10)$. On the other hand, studies also showed that nanostructured surfaces can promote the adsorption of proteins, improve the hydrophilicity and blood compatibility of materials, and greatly promote the biological behavior of osteoblasts $(11,12)$, especially when it forms a hierarchical composite structure with microstructures such as GNS surface obtained by SMAT (13). Qin et al. (14) prepared a micro/ nanostructured surface on a Co-Cr-Mo alloy and the results showed that the cell activity of MC3T3-E1 osteoblasts was significantly improved. In vivo studies by Rezaei et al. (15) showed that the osseointegration strength of zirconia with a meso/micro/nano surface is twice than that of mechanical zirconia at 2 and 4 weeks postoperatively. In addition to the improved surface bioactivity, SMAT also has the advantages of easy realization, low energy consumption, and environmental protection (16). The researchers believe that SMAT technology could be applied as a method to improve the comprehensive properties of metal materials.

To apply the SMAT technology into the orthopedic and dental implant field, in our previous study, Wen et al. fabricated GNS Ti with a nanocrystalline layer of about $30 \mathrm{~nm}$ thick utilizing SMAT (17). Results showed that GNS Ti shows much-improved oxidation kinetics in $\mathrm{H}_{2} \mathrm{O}_{2}$ solution, and more stable oxide thickness compared with coarse grained (CG) Ti. This stable oxide layer has a positive effect on surgical healing indicating that the SMAT could be a possible method to improve the bioactivity of Ti implants. To realize the clinic application, we intend to evaluate the biocompatibility of GNS Ti further comprehensively by evaluating the adhesion, proliferation, and osteogenic differentiation of bone mesenchymal stem cells (BMSCs) in vitro and assessing the osteogenesis and osseointegration in vivo.

In this study, GNS Ti was previously fabricated by the SMAT. In vitro experiments, GNS Ti was co-cultured with BMSCs, and the effects of GNS surface on BMSCs adhesion, proliferation, and osteogenic differentiation were respectively evaluated by scanning electron microscope (SEM), MTT, flow cytometry (FCM), alkaline phosphatase (ALP), and osteocalcin (OCN) detection. In vivo experiments, GNS Ti was implanted into the rabbits' mandible, and the effects of GNS Ti on the osteogenesis and osseointegration were evaluated by Micro CT, histological staining, and the immunohistochemical staining of osteogenic factors bone morphogenetic protein 2 (BMP-2). We hope that this study may lay the foundation for the application of gradient nanostructured Ti implants in the fields of dentistry and orthopedics in the future.

We present the following article in accordance with the ARRIVE reporting checklist (available at http://dx.doi. org/10.21037/atm-20-7588).

\section{Methods}

\section{Sample preparation and characterization}

Ti plate $(6,000 \mathrm{~mm}$ in diameter and $5 \mathrm{~mm}$ in thickness) obtained from the Institute of Metal Research, Chinese Academy of Sciences was used in this research. The Ti plates were double-treated at room temperature for $30 \mathrm{~min}$. During the SMAT process, spherical steel balls with a smooth surface are resonated in a reflecting chamber via vibration of 


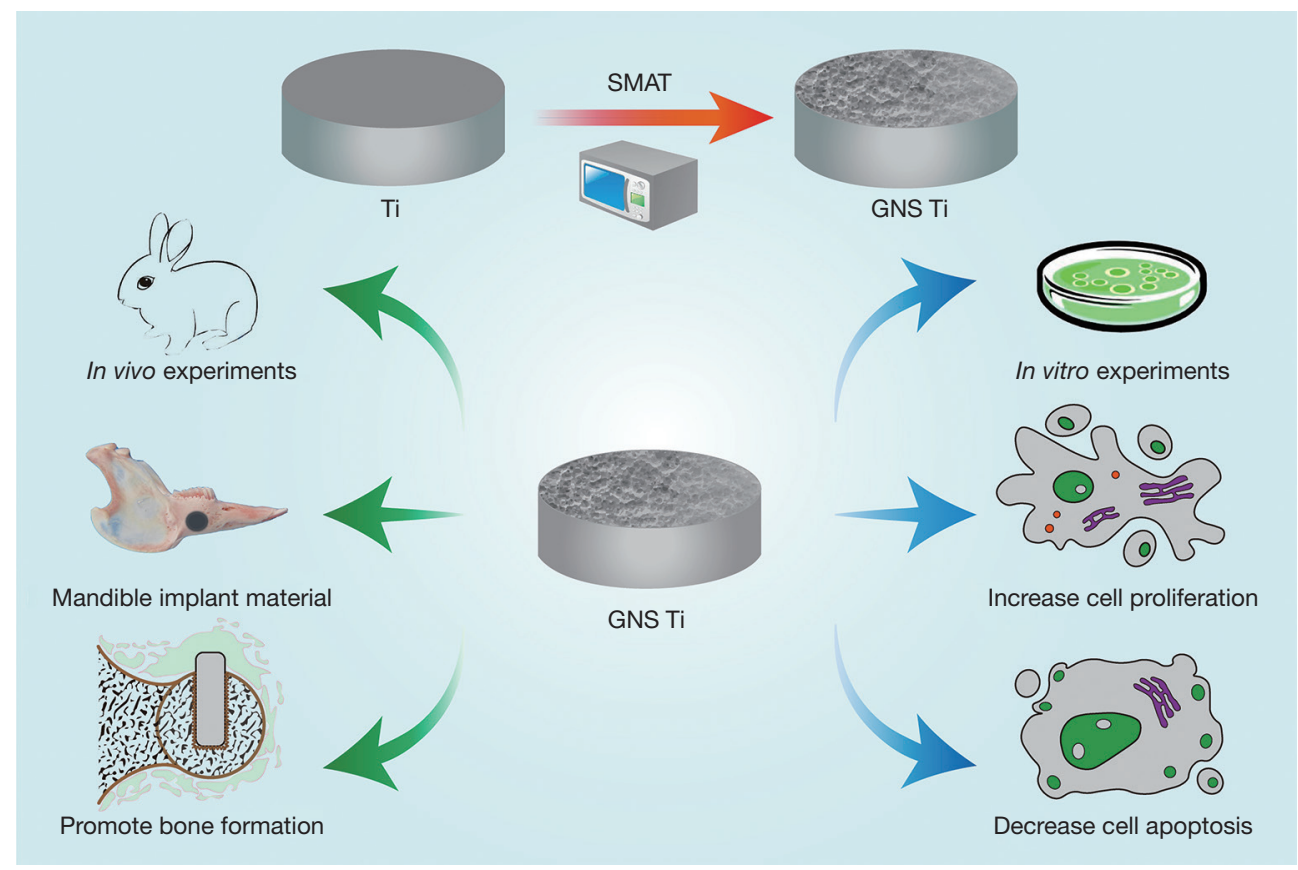

Figure 1 Schematic diagram of the experiment process. SMAT, surface mechanical attrition treatment; GNS Ti, titanium with gradient nanostructured surface.

an ultrasonic transducer. Typically, the ball sizes are $6 \mathrm{~mm}$ in diameter and the vibration frequency of the chamber is $20 \mathrm{kHz}$. After that, a part of the SMAT-treated plates was annealed at $680^{\circ} \mathrm{C}$ for $2 \mathrm{~h}$.

Subsequently, the squares with the size of $\Phi 64 \mathrm{~mm}$ $\times 5 \mathrm{~mm}$ for in vitro experiment and $\Phi 8.0 \mathrm{~mm} \times 2.5 \mathrm{~mm}$ for in vivo experiment were cut from the SMATprocessed and annealed disks, and then rinsed with dilute hydrochloric acid, acetone, ethanol and distilled water for 10 min consecutively, and ultimately dried at $37{ }^{\circ} \mathrm{C}$ for $24 \mathrm{~h}$. Hereafter, the samples treated by SMAT technique are referred to as GNS Ti and the annealed samples are referred to as CG Ti. The surface characterization of GNS Ti and CG Ti was detected by transmission electron microscope (TEM) (JEOL-201, Hitachi, Japan). Schematic diagram of the experiment process was presented in Figure 1.

\section{Cell characterization}

\section{Cell adhesion}

BMSCs were kindly provided by Stem Cell Bank, Chinese Academy of Science. The cells were seeded on the samples placed in 24-well plates at a density of $2 \times 10^{4}$ cells per well. After cultured for $1 \mathrm{~d}$, the samples were washed with PBS for three times. The cells were fixed in $2.5 \%$ glutaraldehyde for $4 \mathrm{~h}$ at $4{ }^{\circ} \mathrm{C}$, dehydrated in graded ethanol series, vacuumdried, and sprayed-coated with gold before observation by the SEM (S3400, Hitachi, Japan).

\section{Cell proliferation}

For the evaluation of cell viability, the cells were cultured on the samples at a density of $1 \times 10^{4}$ viable cells/disc/well for 1, 3, 5, 7 and 14 days, with the MTT Cell Proliferation and Cytotoxicity Assay Kit (MTT, Solarbio, China). MTT assay was precisely performed as described by Kit instructions. Briefly, after culturing in the DMEM/F12 medium for 1, 3, 5, 7 and 14 days, the samples were further cultured for $4 \mathrm{~h}$ in basic medium added with $10 \mu \mathrm{L}$ MTT reagent, and then prepared for optical density (OD) absorbance measurements by using an absorbance microplate reader (Tecan Company, Switzerland) at the wavelength of $490 \mathrm{~nm}$. Data were expressed as absorbance and the growth curve was plotted.

Flow cytometry (FCM) test was carried out to determine the proliferation kinetics of BMSCs on both samples at 5 days. Following the instruction given in the Annexin V-FITC Apoptosis Detection Kit (ANNEXIN V-FITC/PI, Solarbio, China), the samples were successively digested by trypsin and rinsed with PBS, suspended with $1 \mathrm{~mL} 1 \times$ Binding Buffer, 
centrifuged, resuspended with $1 \mathrm{~mL} 1 \times$ Binding Buffer, added with $5 \mu \mathrm{L}$ Annexin V-FITC and $5 \mu \mathrm{L}$ PI. The FCM was performed within $1 \mathrm{~h}$ and the cell proliferation index (PI) was calculated.

\section{Cell differentiation}

When the cells reach $80 \%$ confluency in the medium, the samples were washed and further cultured in the differentiation media at $37^{\circ} \mathrm{C}$ for $3,5,7$ and 14 days. Subsequently, the BMSCs were lysed in PBS at ambient temperature. After centrifugation, the supernatant was used to determine ALP activity with a p-nitrophenyl phosphate assay kit (ALP activity, Solarbio, China), and then prepared for OD absorbance measurements by using an absorbance microplate reader (Tecan Company, Switzerland) at the wavelength of $510 \mathrm{~nm}$.

Besides, the concentration of OCN production of BMSCs was detected by enzyme-linked immunosorbent assay kit (Osteocalcin Human Instant ELISA Kit, Thermo Fisher, America). After the BMSCs were cultured in the osteogenic medium for 7, 10 and 14 days, the supernatant of the medium was collected for the OCN measurements. As chromogenic substrate to horseradish peroxidase antibody, tetramethylbenzidine was used. The concentration of OCN was derived by measuring the OD absorbance value of the mixed solution on a microplate reader (Tecan Company) at the wavelength of $450 \mathrm{~nm}$.

\section{In vivo tests}

\section{Surgical procedures}

Experiments were performed under a project license (No. 2018-13) granted by institutional medical ethics committee of School and Hospital of Stomatology, China Medical University, in compliance with China national or institutional guidelines for the care and use of animals.

The implantation procedure was performed at the Department of Laboratory Animal Science of China Medical University. 18 New Zealand rabbits (3.4 to $4.0 \mathrm{~kg}$ ) obtained from the Qingdao Kangda Biotechnology Co. Ltd. (Qingdao, China) were randomly assigned into two groups, sacrificed at 4,8 , or 12 weeks, with 6 rabbits in each group. The implant surgery was performed under general anesthesia by subcutaneous injection of $20 \%$ urethane at a dose of $5 \mathrm{~mL} / \mathrm{kg}$ body weight and local anesthesia by injection of xylocaine.

An incision paralleled with the inferior border of the mandible was performed at between the posterior border of the centric combination and the anterior border of the masseter muscle, which was about $2.0-2.5 \mathrm{~mm}$ long and $10 \mathrm{~mm}$ above the inferior border of the mandible. After blunt dissection of the underlying tissues, the $\mathrm{WH}$ implant unit was used to prepare the bone defect of $8 \mathrm{~mm}$ in diameter and $2 \mathrm{~mm}$ in depth on the bilateral mandible. During the operation, saline was cooled and cotton swabs were pressed to stop bleeding. The implants were slightly inserted into each side of the bone defects randomly with good primary stability. Postoperatively, the animals were given intramuscular injections of penicillin $(800,000 \mathrm{IU} / \mathrm{mL})$ for 3 days to prevent postoperative infection.

\section{Micro-computed tomography analysis}

Three rabbits were respectively sacrificed at 4,8 , and 12 weeks postoperatively and the implants with $5 \mathrm{~mm}$ surrounding bone tissue were harvested and scanned by micro-CT analysis (Skyscan 1276, Bruker Micro-CT, Kontich, Belgium). The working parameters of CT are set as follows: voltage is $100 \mathrm{kVp}$, the current is $200 \mathrm{~mA}$ with a 360 -degree scan, and ROI was set to be the region from 100 to $1,000 \mathrm{~mm}$ above the $\mathrm{Ti}$ implant-bone. Image reconstruction was performed using the graphics processing unit (GPU)-based reconstruction software, GPU-Nrecon. Data on percent bone volume (BV/ TV: \%), trabecular thickness (Tb.th: $\mathrm{mm}$ ), and trabecular separation (Tb.sp: $\mathrm{mm}$ ) were retrieved from the ROI selected.

\section{Toluidine blue staining}

Three rabbits were sacrificed for undecalcified histological analysis at 4,8 , and 12 weeks postoperatively. The samples were fixed in 4\% paraformaldehyde dehydrated in alcohol series and infiltrated in methyl methacrylate, and finally embedded in polymethyl methacrylate. Bone blocks were sectioned along a parallel to the longitudinal axis of Ti discs with E300CP diamond saw microtome (EXAKT, Germany), obtaining a series of sections and subsequently polished to a thickness of 40-50 $\mu \mathrm{m}$. Four consecutive sections of the central portion of the implant site were obtained for each sample and stained with Toluidine blue staining solution. Histological images were taken by optical microscopic and analyzed with a digital image acquisition system at $4 x$ and $10 \times$ of magnification.

BIC was calculated by dividing the length of the traits of the implant in contact with the bone to the total length of the implant of which the formula was as follows:

$\% \mathrm{BIC}=$ (length of the osseointegration part of the bone-implant interface/length of the whole part of the bone-implant interface) $\times 100 \%$ 


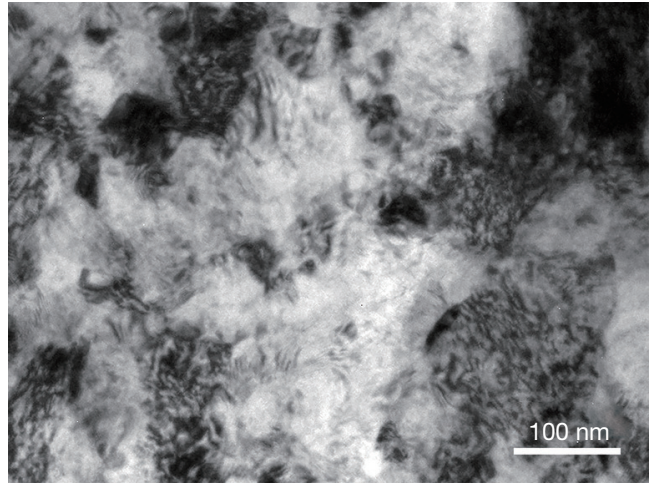

Figure 2 Typical plane-view TEM observation of the GNS Ti. TEM, transmission electron microscope; GNS Ti, titanium with gradient nanostructured surface.

Besides, at all experimental times, the rate at which BIC is formed in the time was calculated as the ratio of BIC percentage per week.

\section{Immunohistochemical staining}

At 4,8 , and 12 weeks after implantation, the rabbits were respectively sacrificed, and the mandibles were collected. For immunohistochemical staining, the specimens were decalcified and embedded in paraffin, cut along the sagittal plane and deparaffinized. After antigens were recovered by $0.05 \%$ parenzyme for $20 \mathrm{~min}$ and blocked by BSA, sections were incubated with primary antibodies (Mouse Anti-BMP-2 antibody, ab6285, Abcam, UK). Then, secondary staining was observed using the secondary antibody (Goat Anti-Mouse, ab205719, Abcam, UK) and 3,3'-diaminobenzidine (DAB, ab64261, Abcam, UK). Microscopic images of representative regions of the stained tissues were obtained using Axioskop microscopy (Olympus IX71, USA). The images were acquired using a ToupCam TP610000A microscope acquisition system. For immunohistochemical analysis, BMP-2 positive expression was shown in brown. The average optical density (AOD) of the positive region was measured using an Image-Pro Plus 6.0 software (Image-Pro Plus, Media Cybernetics, USA) by randomly selecting 3 high-power fields.

\section{Statistical analysis}

All data were expressed as mean \pm standard deviations (SD). Statistical analysis was performed using SPSS 21.0 software (IBM, USA) by Independent Samples $t$-test to compare the data between different groups. Statistical significance was considered at $\mathrm{P}$ value less than 0.05 . At least three samples were used for each test group.

\section{Results}

\section{Surface characterization}

As shown in Figure 2, the nano-scale grains with random crystallographic orientations in the topmost surface layer of the SMAT samples were clearly revealed by TEM.

\section{Cell characterization}

The SEM images of BMSCs on both samples are shown in Figure $3 A$ and $B$. It is observed that BMSCs adhered well on both samples for 1 day. Moreover, compared to CG Ti, BMSCs spread more homogenously and fully on the GNS Ti. Meanwhile, the cells on the GNS Ti were predominantly in the shape of a polygon, with numerous filopodia protruding into the sample surface and connecting neighboring cells.

Figure $3 C$ presents OD absorbance curves obtained by MTT of both samples at different time points to evaluate the proliferation kinetics of BMSCs $(18,19)$. Although the cell densities are at a low level for 1 day on both samples, the proliferation kinetics of the cells on GNS Ti showed a significant promotion during the incubation for 3,5 , and 7 days. Statistical analysis showed that there are significant differences between the two groups in OD values for 3, 5, and 7 days $(\mathrm{P}<0.01)$. And it is worth noted that at 7 days, the proliferation peak of both samples, the OD value of the GNS Ti is $0.812 \pm 0.006$, while it is only $0.517 \pm 0.008$ in CG group.

FCM tests were performed to further confirm the promoted proliferation kinetics of both samples by measuring the cell apoptosis of BMSCs (Figure 3D,E). The result showed that after incubation for 5 days the PI value of the GNS Ti is significantly higher than that of the CG $\mathrm{Ti}(\mathrm{P}<0.01)$. Moreover, it is worth noting that the PI value of the GNS Ti is almost twice of that of the CG Ti.

In order to find out the osteogenic differentiation kinetics of BMSCs on both samples, we measured the ALP activity for 3, 5, 7, and 14 days and OCN activity for 7, 14 days respectively (Figure $3 F, G$ ). An increasing trend of ALP activity with the extension of culture time is observed in both samples. At each time point, the ALP activity of GNS Ti was always higher than that of $\mathrm{CG} \mathrm{Ti}$, and the difference between the two groups is significant in statistics $(\mathrm{P}<0.01)$. Till the last 

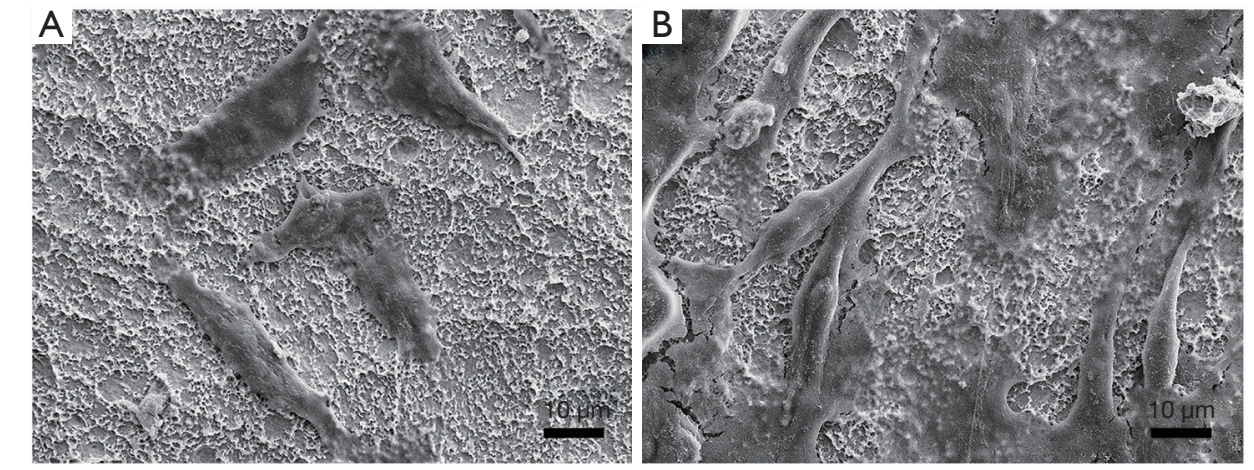

C

D
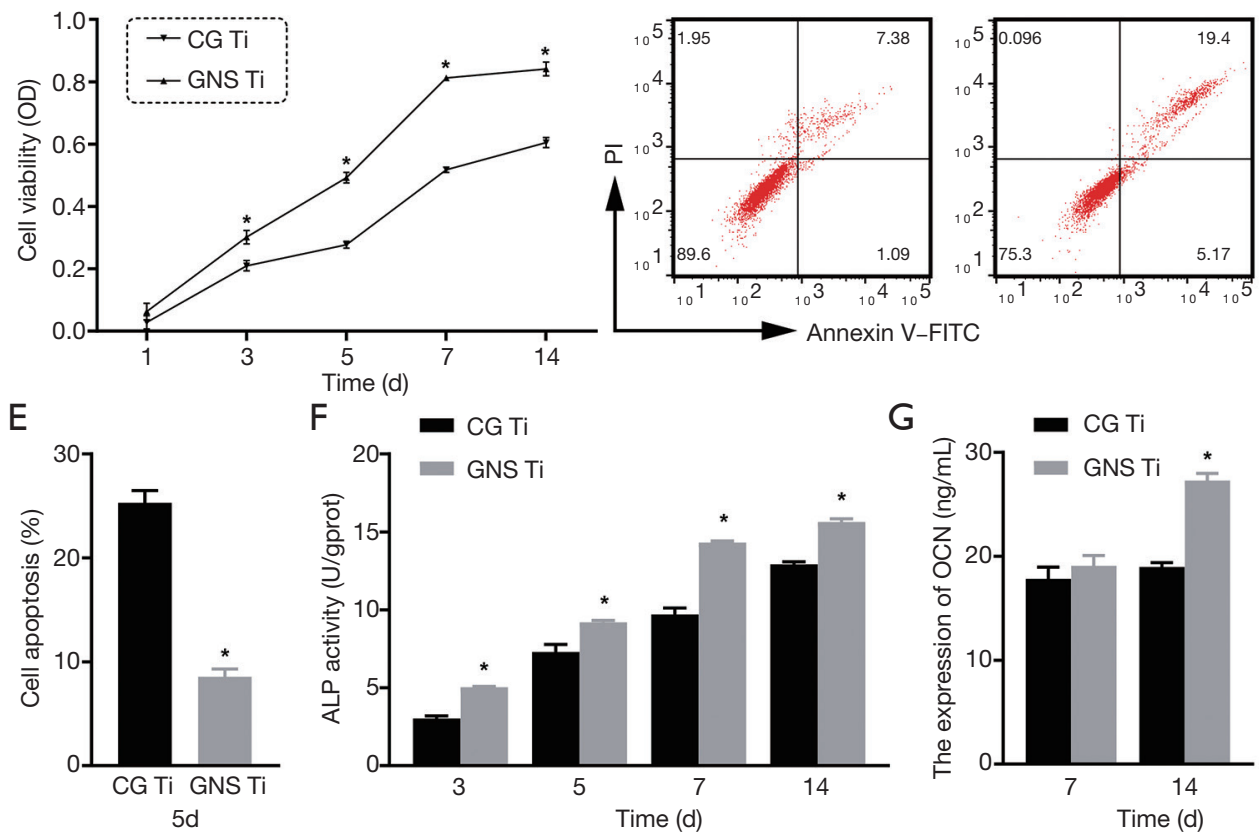

Figure 3 Function of BMSCs on the CG Ti and the GNS Ti samples. SEM morphologies of BMSCs adhering to (A) the CG Ti and (B) the GNS Ti after incubation for $1 \mathrm{~d}$. (C) Proliferation curves of BMSCs on both samples as reflected by measuring OD absorbance values after incubation for 1, 3, 5, 7, and 14 days. (D) Flow cytometry results show the BMSC apoptosis rate for 5 days of incubation. (E) Comparison of cell apoptosis between the CG Ti and GNS Ti for 5 days. Differentiation curves of BMSCs cultured on the CG Ti and the GNS Ti as reflected by measuring (F) ALP activity after incubation for 3, 5, 7 and 14 days and (G) OCN concentration after incubation for 7 and 14 days. Error bars represent means $\pm \mathrm{SD}$ for $\mathrm{n}=3$, * indicates $\mathrm{P}<0.01$. BMSC, bone mesenchymal stem cell; CG Ti, coarse grained titanium; OCN, osteocalcin; OD, optical density.

testing time the ALP activity of the GNS Ti is about $36 \%$ higher than that of the CG Ti. The OCN concentration of GNS Ti is statistical higher than that of CG Ti at 14 days $(\mathrm{P}<0.01)$.

\section{In vivo performance}

\section{General observation}

Eighteen New Zealand rabbits were used in the implantation operation. After implantation surgery the rabbits eat a small amount of food at 3-4 days and eat normally at 5-7 days. There was no obvious inflammation at the implant site (Figure 4), no loosening of the implant, and no soft tissue growth at the implant-bone tissue interface.

\section{Micro-computed tomography analysis}

Micro-CT was used to evaluate new bone formation at the 
A

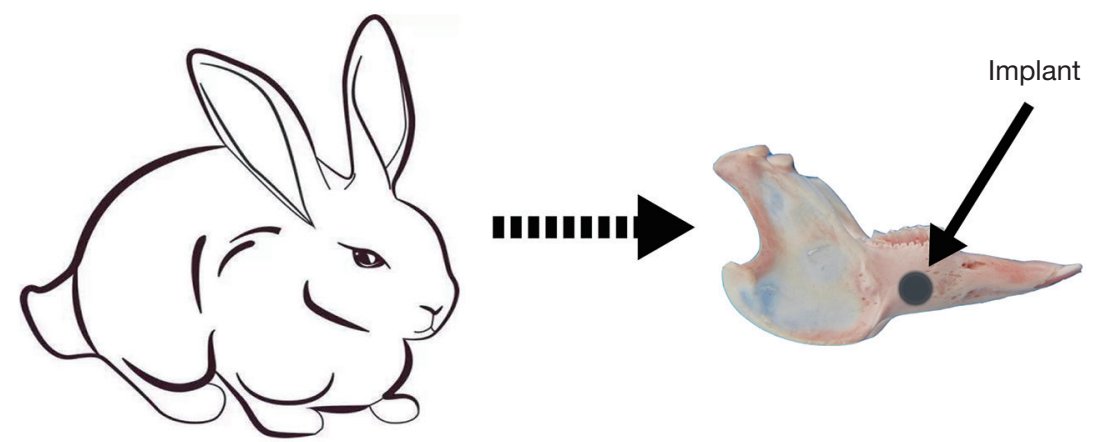

Rabbit

Mandible
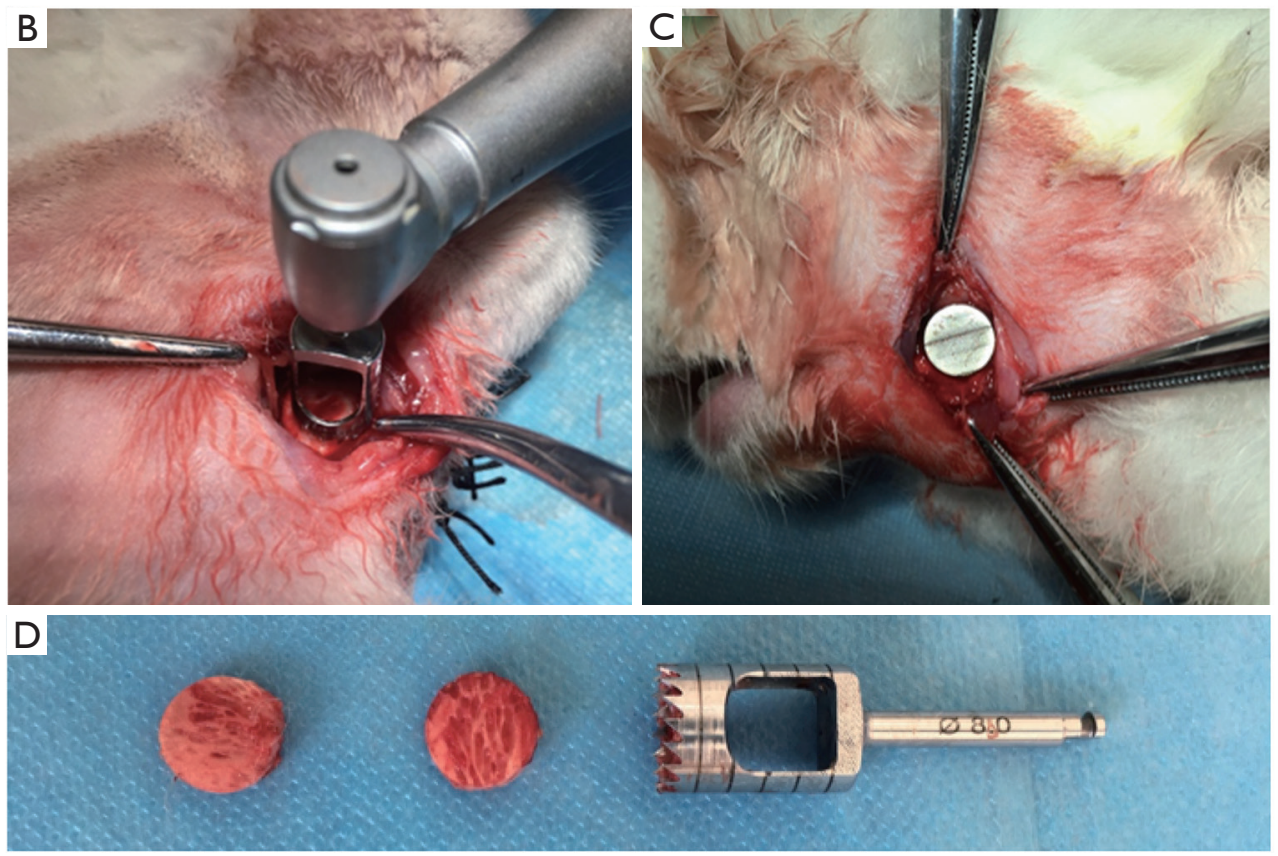

Figure 4 Schematic plot and implantation process of the surgical procedure in rabbit mandible. (A) The schematic plot of the planting site; (B) preparation of mandibular bone defect; (C) bone graft defect of the sample; (D) bone tissue removed by the planter.

bone-implant interface. In general, compared with the CG $\mathrm{Ti}$, the GNS Ti showed significantly more bone formation at 4, 8, and 12 weeks postoperatively. As shown in Figure 5, on the surface of GNS Ti, new bone was observed at 4 weeks postoperatively. As time passed, bone tissue gradually grew along the surface of the implant. At 12 weeks, the bone tissue was in close contact with the implant and the height was uniform and tended to be continuous. In contrast, the amount of bone tissue formed on the CG Ti was less than that on GNS Ti at 4 weeks after the operation. At 12 weeks, the bone tissue on the CG Ti was mostly concentrated on the edge of the material, and the bone formed in the center of the material was little.
The percent bone volume (BV/TV, \%) and trabecular thickness (Tb.th, $\mathrm{mm}$ ) represent the bone volume rate and the thickness of the bone respectively. At 100-1,000 mm exterior to the implant, both parameters of the GNS Ti were significantly higher than that of CG Ti with statistical differences $(\mathrm{P}<0.05)$. The trabecular separation (Tb.sp, $\mu \mathrm{m})$ represents the average distance between the trabecular. It was lower in the GNS Ti group than in CG Ti group with statistical significant difference $(\mathrm{P}<0.05)$.

\section{Histological analysis}

Toluidine blue staining showed that, at 4,8 , and 12 weeks after implantation, the amount of new bone formation on 

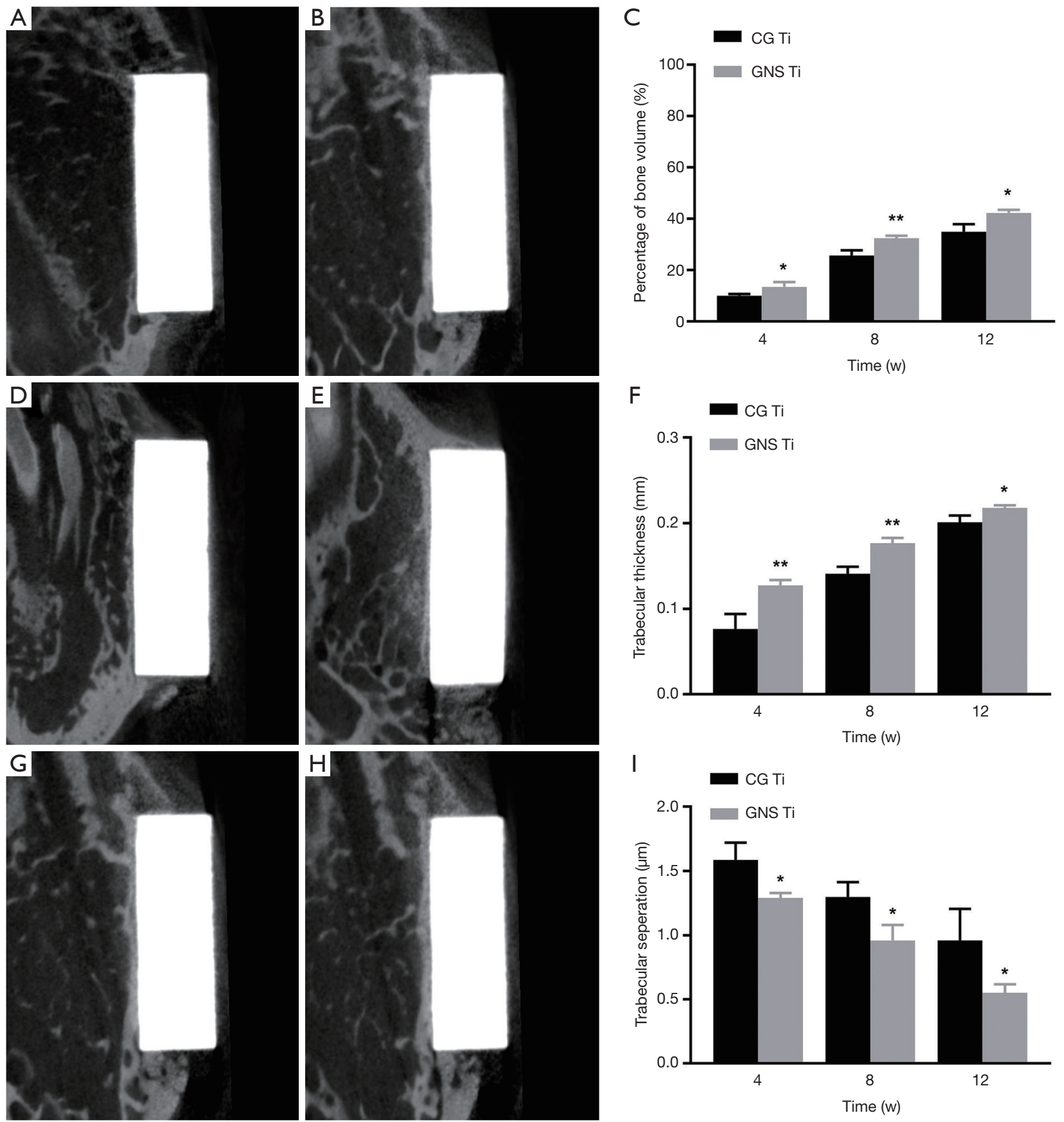

Figure 5 Images and graphs of bone histomorphometric parameters obtained by 3-dimensional reconstructed micro-computed tomography of osseointegration in rabbit mandible. (A,D,G) The CG Ti and (B,E,H) the GNS Ti with the surrounding bone tissue after 4, 8 , and 12 weeks postoperatively. (C) Bone volume fraction (BV/TV), (F) trabecular thickness (Tb.th) and (I) trabecular separation (Tb.sp) of both samples. Data are presented as means \pm SD for $\mathrm{n}=3,{ }^{*}, \mathrm{P}<0.05 ;{ }^{* *}, \mathrm{P}<0.01$. GNS Ti, titanium with gradient nanostructured surface; CG Ti, coarse grained titanium. 

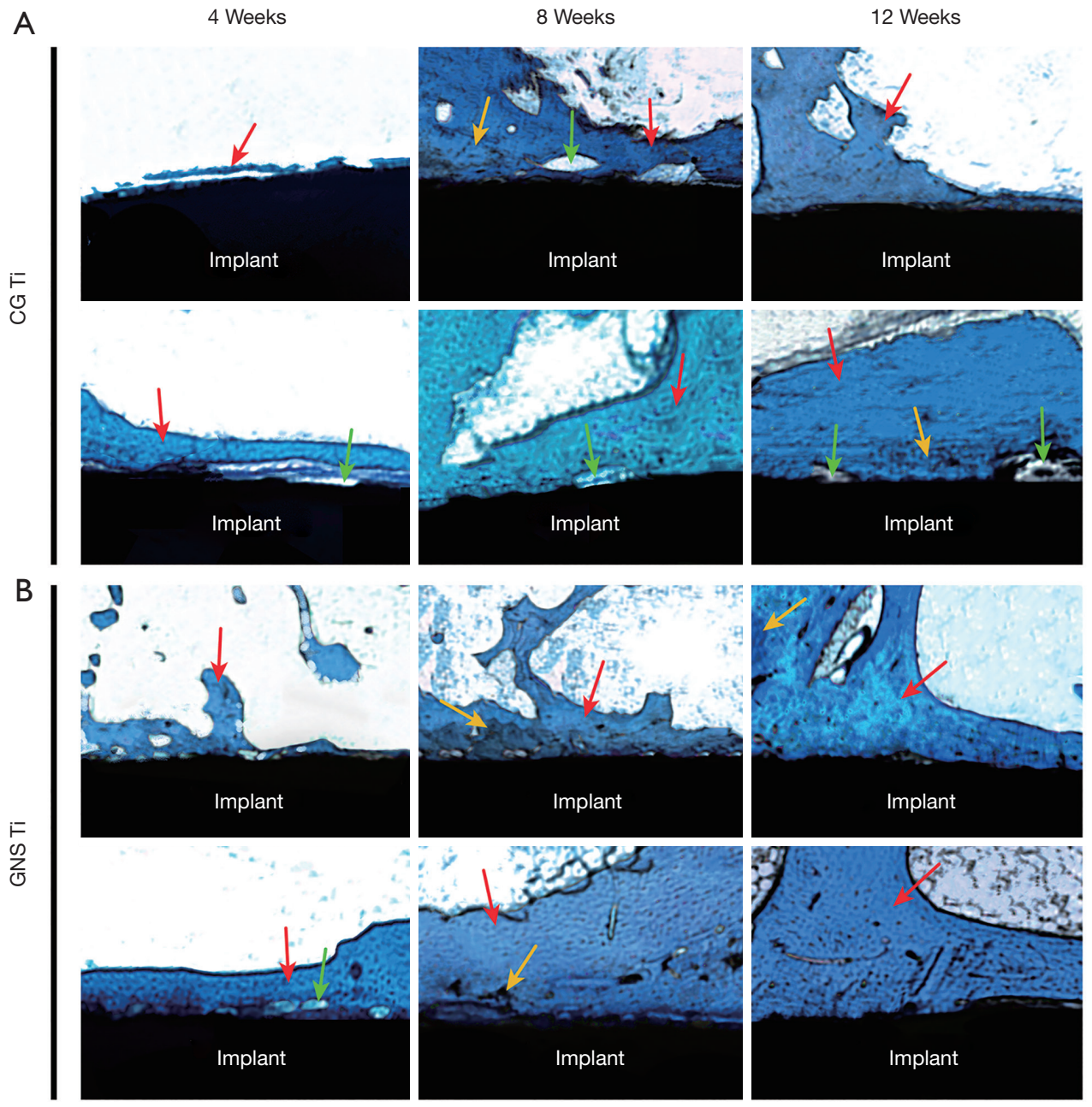

Figure 6 Photomicrography of toluidine blue staining. (A) The CG Ti and (B) the GNS Ti at 4, 8, and 12 weeks postoperatively. New bone tissue, the host bone tissue, and the gap between the implant and the bone tissue are indicated by arrows of red, yellow and green, respectively. GNS Ti, titanium with gradient nanostructured surface; CG Ti, coarse grained titanium.

the GNS Ti was higher than that on the CG Ti (Figure 6). At 4 weeks postoperatively, the bone tissue on the GNS Ti was irregular in shape and extended to the periphery while new bones on the CG $\mathrm{Ti}$ was thinner and had a certain gap with the implant surface. At 8 weeks after surgery, the bone tissue on the GNS Ti continued to grow along the surface and began to form a mature trabecular shape, while the trabeculae around the CG $\mathrm{Ti}$ was relatively sparse. At 12 weeks after surgery, GNS Ti formed a tight bond with surrounding bone tissue, while CG Ti still had gaps between the substrate and the bone tissue.

To compare the trend of new bone formation between two groups, we quantified bone-implant contacts (BIC) value using Image J program (20) (Figure 7). BIC values for the GNS Ti and CG Ti were observed at 4, 8, and 12 weeks $(\mathrm{P}<0.05)$. BIC value of GNS Ti was almost 1.6 times than that for the CG Ti at 8 weeks $(\mathrm{P}<0.01)$.

The immunohistochemical staining of BMP-2 was shown in Figure 8A. A wide range of positive reactions was observed in the mesenchymal stem cells and osteoblasts between the implant and bone tissue of the GNS sample. Figure $8 B$ showed that significantly higher expression of BMP-2 was detected in the GNS group compared with the CG group $(\mathrm{P}<0.01)$ at 4 weeks after implantation. Moreover, as time increasing the expression levels of BMP-2 gradually decreased. At 8 weeks, the expression level of BMP-2 in the GNS group was still higher than that in the $C G$ group $(\mathrm{P}<0.05)$. But at 12 weeks, there was no 


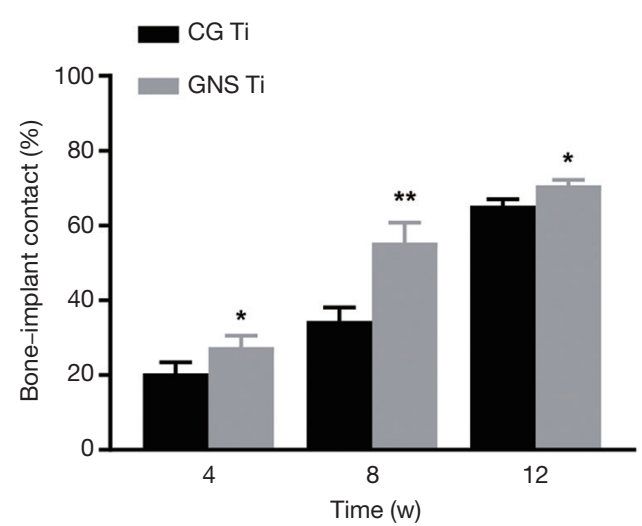

Figure 7 Graphs of values obtained from bone-implant contact analysis. Data are presented as means $\pm \mathrm{SD}$ for $\mathrm{n}=3$, *, $\mathrm{P}<0.05$; **, $\mathrm{P}<0.01$. GNS $\mathrm{Ti}$, titanium with gradient nanostructured surface; CG Ti, coarse grained titanium.

significant difference in the expression of BMP-2 between the two groups $(\mathrm{P}>0.05)$.

\section{Discussion}

Currently, more than $70 \%$ of biomedical implants are made of metal materials. Considering the growing clinical demand, it is necessary to further improve the biological activity of the implant materials and enhance the osseointegration between the materials and surrounding bone tissues $(21,22)$.

SMAT is a promising technology to induce a nanocrystal line surface layer on the metal surface without interface between the base metal and the nanostructure layer (10). This technology could contribute a strong bond between the surface layer and the matrix overcoming the disadvantage of potential peeling risk of traditional coating methods. TEM images of SMAT metals exhibit randomly distributed nano grains on the topmost surface indicating that SMAT is a stable technology $(23,24)$.

In our research, the SMAT Ti (GNS Ti) was cocultured with BMSCs. As one of the most widely studied mesenchymal stem cells, BMSCs have low immunogenicity and high osteogenic differentiation potential, which is very important to the establishment of trabeculae $(25,26)$. The SEM images showed that cells cultured on GNS Ti have better spread and extended morphology than on CG Ti. Cells on GNS Ti extend more uniform than on CG Ti. Moreover, cells grown on GNS Ti is polygonal with many pseudopods protruding into GNS Ti surface and connecting with neighboring cells. However, the morphology of cells cultured on CG Ti is mainly spindle with little pseudopods. This result suggests that the GNS Ti has higher bioactivity and could promote cell spreading and adhesion.

It was also found that the proliferation of BMSCs in the GNS Ti group was significantly enhanced during 3-7 days of culture, mainly manifested in the significant difference in $\mathrm{OD}$ value between the groups at $3-7$ days $(\mathrm{P}<0.01)$. The enhancement of cell proliferation was further confirmed by FCM detection. At day 5, the PI value of the GNS Ti group was about 2.5 times higher than that of the CG Ti group with statistical significance $(\mathrm{P}<0.01)$.

Typically, ALP activity and OCN concentration are markers representing the early stage and the late stage of the osteogenic differentiation process, respectively $(27,28)$. The present study shows that BMSCs cultured on GNS Ti has significant higher ALP activity than cells grown on $\mathrm{CG} \mathrm{Ti}$ at days $3-14(\mathrm{P}<0.01)$. As for $\mathrm{OCN}$ concentration, there is a same expression pattern as that of ALP. The OCN expression in GNS Ti group was significantly up-regulated on days $7-14$, while the OCN concentration in CG Ti is similar in two study period. Studies have shown that the nanostructured surface can directly promote the proliferation and osteogenesis differentiation of mesenchymal stem cells by increasing the levels of osteogenic differentiation markers, such as osterix (OSX), ALP, and OCN $(7,29)$. The up-regulation of the ALP and OLP expression suggests that the GNS surface could promote bone formation by promoting osteogenic differentiation of stem cells. This result is consistent with previous studies (30-32).

Based on the above results, the GNS Ti has great biocompatibility in enhancing the adhesion, proliferation, and osteogenic differentiation of BMSCs.

It is reported that the roughness of biomaterial surface plays a critical role in material biocompatibility (33). In the current research and our previous studies (34), to exclude the impact of surface roughness in material biocompatibility, the control group (CG Ti) is fabricated by SMAT treatment followed by recrystallization annealing treatment making sure a similar roughness between GNS Ti and CG Ti. In our previous research, Wang Wei have found that the roughness of GNS Ti is $1.73 \pm 0.11 \mu \mathrm{m}$ and roughness of CG Ti is $1.82 \pm 0.06 \mu \mathrm{m}$ (31). There is no statistical difference between the two outcomes. Therefore, we could exclude the impact of roughness on the biocompatibility of the two samples. 

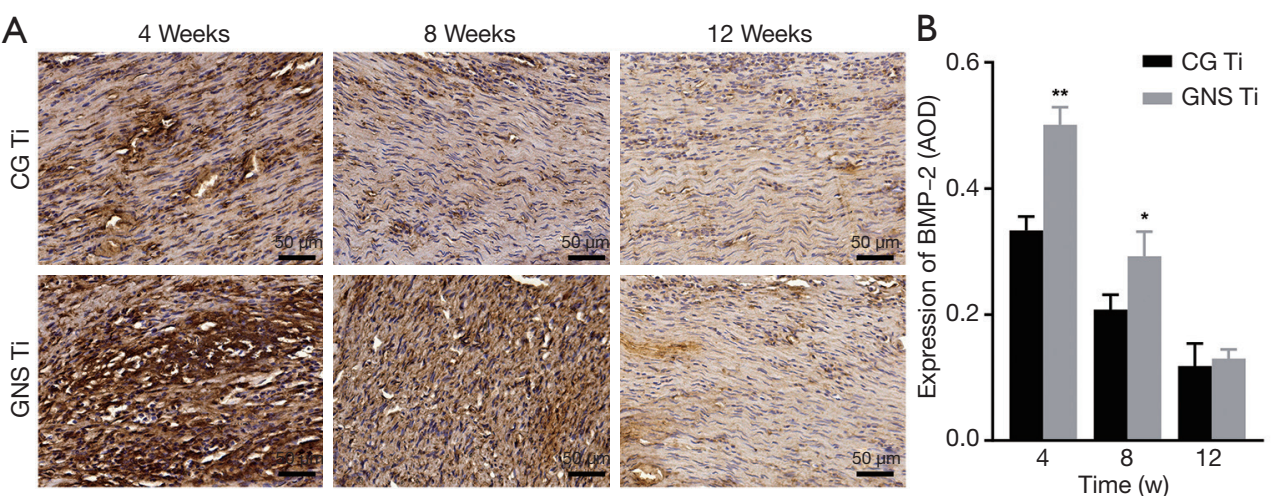

Figure 8 Immunohistochemical analysis of BMP-2 after CG Ti and GNS Ti implantation. (A) BMP-2 expression levels were detected using immunohistochemical evaluation, and positively stained brown cells were seen. (B) Quantitative analysis of new bone formation expressed as BMP-2 levels at 4, 8, and 12 weeks after implantation. Error bars represent means $\pm \mathrm{SD}$ for $\mathrm{n}=3$, *, $\mathrm{P}<0.05$; **, $\mathrm{P}<0.01$. BMP-2, bone morphogenetic protein 2; GNS Ti, titanium with gradient nanostructured surface; CG Ti, coarse grained titanium.

Hydrophilicity of material surface, mechanics of material, and nanostructure of surface have all be proven to have influence on the material biocompatibility. In this research GNS Ti have been found to have higher bioactivity than CG Ti in promoting BMSCs proliferation, adhesion, and osteogenesis differentiation, which could be explained by the better nanostructure, higher hydrophilicity, and better mechanics.

It is reported that the nanoscale surface could promote the cytoskeleton forming and the interfacial tension of membrane receptors and induce the cells to take the surface structure for growth orientation (32). Additionally, cells prefer to adhere, spread, and grow on hydrophilic surfaces. In our previous research, we have measured the hydrophilicity of the two samples by contact angle. We found that the contact angle of GNS $\mathrm{Ti}$ is $53.7^{\circ} \pm 2.4^{\circ}$, while the contact angle of CG Ti is $96.9^{\circ} \pm 3.8^{\circ}$. There is a significant difference between the two groups in contact angle indicating that GNS Ti has high hydrophilicity (34). Therefore, the higher hydrophilicity of GNS Ti is a promoting factor for its higher biocompatibility.

Material mechanics plays a critical role in the material application. In fact, surface properties are determining factors in material mechanics (wear, corrosion, fatigue etc.). Materials with low wear resistance could release ions into surrounding tissues causing inflammation and allergy (35). Wen et al. have found that GNS Ti has lower friction coefficient than CG $\mathrm{Ti}$ (36). It indicates that GNS Ti will perform stable in oral environment. Moreover, the strength of GNS Ti is proven to be enhanced by its nanocrystalline surface compared with CG Ti (37). The increased mechanic properties of GNS Ti is a reason for its better biocompatibility.

It is valuable to investigate the promoting osteogenesis ability of GNS Ti further. Chen et al. have cultured osteoblast cells on Ti6Al4V surface. Over a culture period of 35 days, discrete bone-like nodules could be seen by naked eyes on the Ti6Al4V surface. After analyzed by environmental scanning electron microscopy (ESEM), atomic force microscopy (AFM) and energy dispersive $\mathrm{X}$-ray (EDX), the outcome showed that the bone-like nodules have similar structure and $\mathrm{Ca} / \mathrm{P}$ ratio to natural bone (38). However, it is unclear the facilitating osteogenesis ability of GNS Ti in this aspect. In the future, we will prolong the experimental period to further study the mechanics of bone nodules formed on GNS Ti surface to investigate the bone formation ability of GNS Ti deeply.

Based on the above in vitro studies, we further investigated the promoting osteogenesis ability of GNS Ti in vivo by implanting GNS Ti and CG Ti into the New Zealand rabbit mandibular. Micro CT scanning showed that at 12 weeks after surgery, highly uniform bone tissue was formed on the surface of GNS Ti, while the bone tissue on the CG $\mathrm{Ti}$ is limited on the edge of the material. Morphological analysis showed that BV/TV and Tb.th in the GNS Ti group were significantly higher than CG $\mathrm{Ti}$ $(\mathrm{P}<0.05)$, and $\mathrm{Tb}$.sp was significantly lower $(\mathrm{P}<0.05)$ than CG $\mathrm{Ti}$ at 4,8 , and 12 weeks. It indicates that the bone binding strength in the GNS Ti group was significantly higher than that of the CG Ti. This outcome was consistent with the research of Johansson et al. (39). Toluidine blue staining further confirmed the results of Micro CT as 
shown in Figure 5. At 12 weeks postoperatively, bone tissue on the surface of GNS Ti had formed a close bond with the material, while there was still a gap between CG Ti and bone tissue. By analyzing the BIC ratio of the two groups, it was found that BIC in the GNS Ti group was significantly higher than that in the CG Ti group $(\mathrm{P}<0.05)$. Especially at 8 weeks after the operation, BIC in the GNS Ti group was about 1.6 times higher than that in the CG Ti group $(\mathrm{P}<0.01)$.

Bone tissue parameters and the BIC analysis were mutually verified, indicating that GNS Ti could accelerate bone formation and improve the microstructure of bone tissue, and promote the implant-bone binding. Referring to the concept of osseointegration, the increased implant-bone contact and promoted bone microstructure of the GNS $\mathrm{Ti}$ is conducive to the long-term stability of the material. In addition, it should be pointed out that the differences between GNS Ti and CG Ti mainly occurred at 4-8 weeks after the surgery, which can be considered as the main effect period of GNS Ti in vivo.

The ability of implant materials with nano/micro surface in promoting osseointegration is proved by other researches. Zhu et al. (40) implanted bioceramics with nano/micro surface into the long bone defect of Beagle dogs, and tight osseointegration was observed at 12 weeks after surgery, while there were still gaps between the implant surface and bone in the control group. In addition, Liu et al. (41) implanted strontium-loaded micro/nano Ti into the mandible of ovariectomized sheep, and the results showed the BV/TV and the Tb.N of nano/micro surfaces are significantly higher and Tb.sp is lower than those in other groups. The improved bone tissue morphology parameters in the animal model indicate that GNS Ti may be further applied in the field of dental implants.

The bone reconstruction process is influenced by systemic factors. As receptors, the biological activity of bone cells is largely regulated by calcitonin, estrogen, growth factors, and cytokines (42). Bone morphogenetic protein $(\mathrm{BMP})$ is an early signaling molecule for bone formation $(43,44)$. Among them, BMP-2 has the strongest osteogenic activity (45), which can not only induce bone formation in stem cells, but also recruit stromal cells (46). In this experiment, the expression of BMP-2 at the implantbone tissue interface was detected by immunohistochemical analysis. The results showed that at 4 weeks, the expression intensity of BMP-2 in the GNS group was significantly stronger than that in the CG group, with a statistically significant difference $(\mathrm{P}<0.01)$. However, the expression levels of BMP-2 of the two groups showed a decreasing trend over time. At 8 weeks, the difference between the two groups was statistically significant $(\mathrm{P}<0.05)$, but at 12 weeks, there was no statistically significance $(\mathrm{P}>0.05)$. The reason of this phenomenon is that as new bone maturing, the role of BMP-2 in bone metabolism diminishes and BMP-2 is stored in calcified substrates (47).

In summary, through in vitro and in vivo experiments, we observed the effects of GNS Ti and CG Ti on the adhesion, proliferation, and differentiation of BMSCs, as well as the effects on the osteogenesis and osseointegration. In vitro experiments showed that GNS Ti could significantly promoted the adhesion, proliferation, and osteogenic differentiation of BMSCs compared with CG Ti. In vivo experiments showed that GNS Ti has higher ability in enhancing osteogenesis and osseointegration than CG Ti.

\section{Conclusions}

Gradient nanostructured Ti fabricated by SMAT has high biocompatibility in promoting bone formation. In vitro experiment showed that GNS Ti significantly improved the proliferation and differentiation of BMSCs compared with CG Ti. In vivo experiment showed that GNS Ti promoted osteogenesis and osseointegration. This study indicated that the gradient nanostructured Ti material may be applied as dental implants in the future.

\section{Acknowledgments}

Funding: This study was supported by National Natural Science Foundation of China (No. 81970980), Liaoning Provincial Key Research Plan Guidance Project (No. 2018225078), Liaoning Provincial Natural Science Foundation Guidance Project (No. 2019-ZD-0749), Central Government of Liaoning Province to Guide Local Science and Technology Development Project (No. 2017108001), Shenyang Major Scientific and Technological Innovation Research and Development Plan (No. 19-1124-027), Shenyang Young and Middle-aged Technological Innovation Talent Plan (No. RC200060).

\section{Footnote}

Reporting Checklist: The authors have completed the ARRIVE reporting checklist. Available at http://dx.doi. org/10.21037/atm-20-7588 
Data Sharing Statement: Available at http://dx.doi. org/10.21037/atm-20-7588

Conflicts of Interest: All authors have completed the ICMJE uniform disclosure form (available at http://dx.doi. org/10.21037/atm-20-7588). The authors have no conflicts of interest to declare.

Ethical Statement: The authors are accountable for all aspects of the work in ensuring that questions related to the accuracy or integrity of any part of the work are appropriately investigated and resolved. Experiments were performed under a project license (No. 2018-13) granted by institutional medical ethics committee of School and Hospital of Stomatology, China Medical University, in compliance with China national or institutional guidelines for the care and use of animals.

Open Access Statement: This is an Open Access article distributed in accordance with the Creative Commons Attribution-NonCommercial-NoDerivs 4.0 International License (CC BY-NC-ND 4.0), which permits the noncommercial replication and distribution of the article with the strict proviso that no changes or edits are made and the original work is properly cited (including links to both the formal publication through the relevant DOI and the license). See: https://creativecommons.org/licenses/by-nc-nd/4.0/.

\section{References}

1. Li Y, Yang C, Zhao H, et al. New Developments of TiBased Alloys for Biomedical Applications. Materials (Basel) 2014;7:1709-800.

2. Karazisis D, Ballo AM, Petronis S, et al. The role of well-defined nanotopography of titanium implants on osseointegration: cellular and molecular events in vivo. Int J Nanomedicine 2016;11:1367-82.

3. Mavrogenis AF, Dimitriou R, Parvizi J, et al. Biology of implant osseointegration. J Musculoskelet Neuronal Interact 2009;9:61-71.

4. Zhao Q, Yi L, Jiang L, et al. Osteogenic activity and antibacterial ability on titanium surfaces modified with magnesium-doped titanium dioxide coating. Nanomedicine (Lond) 2019;14:1109-33.

5. Lu SD, Wang ZB, Lu K. Strain-induced Microstructure Refinement in a Tool Steel Subjected to Surface Mechanical Attrition Treatment. J Mater Sci Mater Med 2010;26:258-63.
6. Li N, Wang N. The effect of duplex Surface mechanical attrition and nitriding treatment on corrosion resistance of stainless steel 316L. Sci Rep 2018;8:8454.

7. Liu X, Song X, Zhang P, et al. Effects of nano tantalum implants on inducing osteoblast proliferation and differentiation. Exp Ther Med 2016;12:3541-4.

8. Du H, An Y, Zhang X, et al. Hydroxyapatite (HA) Modified Nanocoating Enhancement on AZ31 Mg Alloy by Combined Surface Mechanical Attrition Treatment and Electrochemical Deposition Approach. J Nanosci Nanotechnol 2019;19:810-8.

9. Li N, Li YD, Li YX, et al. Effect of surface mechanical attrition treatment on biodegradable $\mathrm{Mg}-1 \mathrm{Ca}$ alloy. Mater Sci Eng C Mater Biol Appl 2014;35:314-21.

10. Guo HY, Xia M, Chan LC, et al. Nanostructured laminar tungsten alloy with improved ductility by surface mechanical attrition treatment. Sci Rep 2017;7:1351.

11. Goldman M, Juodzbalys G, Vilkinis V. Titanium surfaces with nanostructures influence on osteoblasts proliferation: a systematic review. J Oral Maxillofac Res 2014;5:e1.

12. Kopf BS, Ruch S, Berner S, et al. The role of nanostructures and hydrophilicity in osseointegration: Invitro protein-adsorption and blood-interaction studies. J Biomed Mater Res A 2015;103:2661-72.

13. Huang Q, Elkhooly TA, Liu X, et al. Effects of hierarchical micro/nano-topographies on the morphology, proliferation and differentiation of osteoblast-like cells. Colloids Surf B Biointerfaces 2016;145:37-45.

14. Qin $\mathrm{L}, \mathrm{Wu} \mathrm{H}$, Guo J, et al. Fabricating hierarchical micro and nano structures on implantable Co-Cr-Mo alloy for tissue engineering by one-step laser ablation. Colloids Surf B Biointerfaces 2018;161:628-35.

15. Rezaei NM, Hasegawa M, Ishijima M, et al. Biological and osseointegration capabilities of hierarchically (meso-/micro-/nano-scale) roughened zirconia. Int J Nanomedicine 2018;13:3381-95.

16. Zhu LH, Guan YJ, Wang YJ, et al. Influence of process parameters of ultrasonic shot peening on surface nanocrystallization and hardness of pure titanium. International Journal of Advanced Manufacturing Technology 2017;89:1451-68.

17. Wen M, Gu JF, Liu G, et al. Surface evolution of a gradient structured $\mathrm{Ti}$ in hydrogen peroxide solution. Appl Surf Sci 2008;254:2905-10.

18. Chiang CY, Chiou SH, Yang WE, et al. Formation of $\mathrm{TiO}(2)$ nano-network on titanium surface increases the human cell growth. Dent Mater 2009;25:1022-9.

19. Wen M, Wen C, Hodgson P, et al. Improvement of 
the biomedical properties of titanium using SMAT and thermal oxidation. Colloids Surf B Biointerfaces 2014;116:658-65.

20. Franchi M, Bacchelli B, Giavaresi G, et al. Influence of different implant surfaces on peri-implant osteogenesis: histomorphometric analysis in sheep. J Periodontol 2007;78:879-88.

21. de Vasconcellos LM, Oliveira FN, Leite Dde O, et al. Novel production method of porous surface Ti samples for biomedical application. J Mater Sci Mater Med 2012;23:357-64.

22. Niinomi M, Nakai M, Hieda J. Development of new metallic alloys for biomedical applications. Acta Biomater 2012;8:3888-903.

23. Mordyuk BN, Prokopenko GI. Fatigue life improvement of alpha-titanium by novel ultrasonically assisted technique. Materials Science and Engineering: A 2006;437:396-405.

24. Zhang HW, Hei ZK, Liu G, et al. Formation of nanostructured surface layer on AISI 304 stainless steel by means of surface mechanical attrition treatment. Acta Materialia 2003;51:1871-81.

25. Bianco P, Robey PG, Simmons PJ. Mesenchymal stem cells: revisiting history, concepts, and assays. Cell Stem Cell 2008;2:313-9.

26. Deng $W$, Obrocka $M$, Fischer I, et al. In vitro differentiation of human marrow stromal cells into early progenitors of neural cells by conditions that increase intracellular cyclic AMP. Biochem Biophys Res Commun 2001;282:148-52.

27. Bang SM, Moon HJ, Kwon YD, et al. Osteoblastic and osteoclastic differentiation on SLA and hydrophilic modified SLA titanium surfaces. Clin Oral Implants Res 2014;25:831-7.

28. Zajdel A, Kalucka M, Kokoszka-Mikolaj E, et al. Osteogenic differentiation of human mesenchymal stem cells from adipose tissue and Wharton's jelly of the umbilical cord. Acta Biochim Pol 2017;64:365-9.

29. Wang J, An Y, Li F, et al. The effects of pulsed electromagnetic field on the functions of osteoblasts on implant surfaces with different topographies. Acta Biomater 2014;10:975-85.

30. Gittens RA, McLachlan T, Olivares-Navarrete R, et al. The effects of combined micron-/submicron-scale surface roughness and nanoscale features on cell proliferation and differentiation. Biomaterials 2011;32:3395-403.

31. Wang W, Wang Z, Fu Y, et al. Improved osteogenic differentiation of human amniotic mesenchymal stem cells on gradient nanostructured Ti surface. J Biomed Mater Res A 2020;108:1824-33.

32. Zhang Y, Luo R, Tan J, et al. Osteoblast behaviors on titania nanotube and mesopore layers. Regen Biomater 2017;4:81-7.

33. Martinez MAF, Balderrama IF, Karam P, et al. Surface roughness of titanium disks influences the adhesion, proliferation and differentiation of osteogenic properties derived from human. Int J Implant Dent 2020;6:46.

34. Luo X, Liang C, Li N, et al. Effect of Gradient Nanostructured Ti on Behaviours of MG63 Cells In Vitro. J Nanomater 2020. doi: 10.1155/2020/9480537.

35. El Sawy AA, Shaarawy MA. Evaluation of metal ion release from Ti6Al4V and Co-Cr-Mo casting alloys: in vivo and in vitro study. J Prosthodont 2014;23:89-97.

36. Wen M, Wen C, Hodgson PD, et al. Tribological Behaviour of Pure Ti with a Nanocrystalline Surface Layer Under Different Loads. Tribology Letters 2012;45:59-66.

37. Wen M, Liu G, Gu JF, et al. The tensile properties of titanium processed by surface mechanical attrition treatment. Surface \& Coatings Technology 2008;202:4728-33.

38. Chen J, Birch MA, Bull SJ. Nanomechanical characterization of tissue engineered bone grown on titanium alloy in vitro. J Mater Sci Mater Med 2010;21:277-82.

39. Johansson P, Jimbo R, Kozai Y, et al. Nanosized Hydroxyapatite Coating on PEEK Implants Enhances Early Bone Formation: A Histological and ThreeDimensional Investigation in Rabbit Bone. Materials (Basel) 2015;8:3815-30.

40. Zhu Y, Zhang K, Zhao R, et al. Bone regeneration with micro/nano hybrid-structured biphasic calcium phosphate bioceramics at segmental bone defect and the induced immunoregulation of MSCs. Biomaterials 2017;147:133-44.

41. Liu F, Li Y, Liang J, et al. Effects of micro/nano strontium-loaded surface implants on osseointegration in ovariectomized sheep. Clin Implant Dent Relat Res 2019;21:377-85.

42. Florencio-Silva R, Sasso GR, Sasso-Cerri E, et al. Biology of Bone Tissue: Structure, Function, and Factors That Influence Bone Cells. Biomed Res Int 2015;2015:421746.

43. Raschke MJ, Schmidmaier G. Biological coating of implants in trauma and orthopedic surgery. Unfallchirurg 2004;107:653-63.

44. Tang W, Lin D, Yu Y, et al. Bioinspired trimodal macro/ micro/nano-porous scaffolds loading rhBMP-2 for complete regeneration of critical size bone defect. Acta Biomater 2016;32:309-23. 
45. Gothard D, Smith EL, Kanczler JM, et al. Tissue engineered bone using select growth factors: A comprehensive review of animal studies and clinical translation studies in man. Eur Cell Mater 2014;28:166207; discussion 207-8.

46. Mishima Y, Lotz M. Chemotaxis of human articular

Cite this article as: Cao NJ, Zhu YH, Gao F, Liang C, Wang ZB, Zhang Y, Hao CP, Wang W. Gradient nanostructured titanium stimulates cell responses in vitro and enhances osseointegration in vivo. Ann Transl Med 2021;9(7):531. doi: 10.21037/atm-20-7588 chondrocytes and mesenchymal stem cells. J Orthop Res 2008;26:1407-12.

47. Andreas K, Sittinger M, Ringe J. Toward in situ tissue engineering: chemokine-guided stem cell recruitment. Trends Biotechnol 2014;32:483-92. 